\title{
Morphology and Hemodynamic Characteristics of Internal Jugular Vein Hypoplasia and Relation with Cerebral Venous Sinus Stenosis
}

\author{
Lingyun Jia, MD ${ }^{a, b}$, Yang Hua, MD ${ }^{a, b, *}$, Xunming Ji, MD ${ }^{c}$, Kaiyuan Zhang, MD ${ }^{d}$, Yu Tang, MD ${ }^{a, b}$ \\ ${ }^{a}$ Department of Vascular Ultrasonography, Xuanwu Hospital, Capital Medical University, Beijing, China $;{ }^{b}$ Center of Vascular \\ Ultrasonography, Beijing Institute of Brain Disorders, Beijing, China; ${ }^{c}$ Department of Neurology, Xuanwu Hospital, Capital Medical \\ University, Beijing, China; ${ }^{d}$ Department of Radiology, Xuanwu Hospital, Capital Medical University, Beijing, China \\ Received April 15, 2019; revision received April 30; accepted May 4.
}

Objective: The characteristics of Internal jugular vein (IJV) morphology and hemodynamics of IJV hypoplasia have not been well illustrated.

\begin{abstract}
Methods: Seventy-three cases with IJV hypoplasia diagnosed by MR and/or CT venous angiography and 126 healthy control were recruited. Ultrasound was performed to examine the J1-J3 segments of IJV. The diameter and mean flow volume (FVm) of bilateral IJV were compared. The linear regression of bilateral diameter ratio and FVm ratio were analyzed. The optimal cutoff values of diameter and flow volume of different segment of IJVs were determined by receiver operating characteristic (ROC) analysis. Furthermore, the correlation between the IJV hypoplasia and cerebral venous sinus stenosis were analyzed.

Results: There were $91.8 \%$ (67/73) cases with left IJV hypoplasia. The diameter and FVm of hypoplasia IJV were lower than the contralateral side $(P<0.001)$. The bilateral J1 diameter ratio was linear correlation with the FVm ratio, with a coefficient 0.720 . The optimal cut-off diameter ratio of J1-J3 hypoplasia/dominant side were $0.70,0.80$ and 0.75 respectively and the optimal cutoff FVm ratio of three segments were all 0.50 . The side of IJV hypoplasia was highly correlated with the side of transverse sinus and/or sigmoid sinus stenosis with an overall coincidence rate of $68.5 \%$.
\end{abstract}

Conclusion: The left IJV was vulnerable for hypoplasia. IJV hypoplasia was correlated with ipsilateral cerebral venous sinus stenosis. Ultrasound is a reliable modality for evaluating IJV hypoplasia.

Key words: Internal jugular vein; Hypoplasia; Ultrasound; Diameter; Flow volume; Cerebral venous sinus

Advanced Ultrasound in Diagnosis and Therapy 2019;02:035-040

DOI: $10.37015 / A U D T .2019 .190807$

$\mathrm{R}$ ecently, accumulating evidences have suggested that cerebral venous (sinus) system not only reserves and drainages the cerebral blood flow but also maintains the cerebral perfusion, regulates the cerebrospinal fluid and adjusts the intracranial pressure [1]. Internal jugular veins (IJV) are the main outflow pathway of cerebral venous sinuses. Several studies have demonstrated that the IJV abnormalities were related with multiple neurological diseases, such as cerebral venous sinus thrombosis, idiopathic intracranial hypertension, transient global amnesia, transient monocular blindness, and multiple sclerosis [2-6]. IJV hypoplasia was one type of IJV abnormalities. Ultrasound is the modality for screening the morphology and hemodynamics alteration of IJV [2]. While, there is few reports on the morphology and hemodynamics of IJV hypoplasia evaluated by ultrasound. In this study, with healthy individuals as control, the characteristics of venous morphology and

* Corresponding author: Department of Vascular Ultrasonography, Xuanwu Hospital, Capital Medical University, No. 45 Changchun Road, Xuanwu Hospital, Beijing 100053, China; Center of Vascular Ultrasonography, Beijing Institute of Brain Disorders, Beijing, China.

e-mail:dryanghua99@163.com 
hemodynamics were evaluated by ultrasound in patients with IJV hypoplasia and its relationship with cerebral venous sinus stenosis was further investigated.

\section{Patients and Methods}

\section{Patients}

This study protocol was approved by the Institutional Review Board. From October 2017 to June 2018, 73 patients (study group) who were with symptoms of headache, tinnitus, tinnitus cerebri and et al, diagnosed as unilateral IJV hypoplasia by MR venous angiography (MRV) and/or CT venous angiography (CTV) in our institution were recruited. In addition, these patients were categorized as two subgroups: combined with ( $n=50$ cases) or without ( $n=23$ cases) cerebral venous sinus stenosis. At the same time, 126 healthy individuals (control group) were selected as control.

Patients' inclusion criteria: (1) patients with age 1880 years old, (2) patients with the symptoms of headache, tinnitus, tinnitus cerebri and et al. and clinic suspected with cerebral venous insufficiency, (3) MRV and/or CTV showed the unilateral IJV hypoplasia. Exclusion criterial: (1) cerebral venous sinus thrombosis, (2) IJV thrombosis, anomalous valve, (3) congenital heart disease, atrial fibrillation, and cardiac dysfunction, (4) extracranial and intracranial arteries with moderate, severe stenosis or occlusion detected by carotid artery ultrasound and transcranial color-coded sonography (TCCS), (5) IJV cannulation, (6) artery-venous fistula at the upper limb for dialysis, (7) tinnitus and tinnitus cerebri with definite etiology, such as otogenic disease.

Control inclusion criterial: (1) age 18-80 years old. (2) Without symptoms of headache, tinnitus and tinnitus cerebri. (3) Without hypertension or history of cerebrovascular or heart diseases. (4) Heart morphology and function were normal detected by echocardiography. (5) Bilateral IJV and cerebral venous sinus were symmetrical evaluated by MRV and/or CTV.

\section{IJV ultrasonography}

Hitachi Ascendus ultrasound system (Hitachi, Inc., Tokyo, Japan) with a $4-8 \mathrm{MHz}$ microcurvilinear array probe was used to examine the IJV. The examinations were performed by experienced sonographers (with more than 10 years of experiences) according to the protocol we previously reported [2]. The diameters and hemodynamics parameters of the following three segments of IJV were measured: J1 (the level of IJV influx into the innominate vein), J2 (the level at which the superior thyroid vein influx into IJV) and J3 segment (the IJV level representing for the bifurcation level of common carotid artery). The parameters included: (1) the maximum diameters and cross-section areas at the endexpiration during smooth breath period. (2) Under the pulsed wave doppler mode, through auto-enveloping five "M" shape spectrums above the baseline and selecting one of the stable phase with the maximum velocity, then, the maximum, minimal velocity (Vmax and Vmin) and the mean flow volume $(\mathrm{FVm})$ were measured.

\section{$M R V$ and $C T V$ examinations}

MagnetomVerio3.0 T MRI station (Siemens Inc., Germany) with head and neck 8-channel coils was used to perform MRV examination. The screening ranges were from aorta arch to the top of head. The magnevist (Bayer pharmaceutical Inc., Levekusen, Germany) were used as the contrast. Before injection of the contrast, routine scan and triple-phase contrast-enhanced scan were performed. Then 3-D head and neck veins images were reconstructed by the maximum intensity projection (MIP) processing [7]. In patients with contraindication for MRV examination (such as with metal materials), CTV examination was performed to evaluated the IJV and cerebral venous sinuses. IJV hypoplasia was defined as: the diameter of one side of IJV was obvious smaller than the other side of IJV detected by MRV and/or CTV (the whole length of one side IJV was moderate to severe stenosis) [8]. The transverse sinus and/or sigmoid sinus hypoplasia was defined as the difference of bilateral diameters of sinuses was $>50 \%[9]$.

\section{Statistical analysis}

The Statistical Package for Social Sciences (SPSS version 22.0) software was used for the statistical analysis. Numerical values with normal distribution were shown as mean \pm SD and with non-normal distribution shown as median (interquartile range). Paired t test was used to compare the parameters of bilateral IJV in both study and control group. Two-independent sample $t$ test was used to compare the parameters between the study and control group and between the two subgroups. Linear regression was used to analyze the correlation between the parameter of diameter ratio and $\mathrm{FVm}$ ratio and a linear regression equation was established. The optimal cut-off values of diameter and flow volumes of different segment of IJVs were determined by receiver operating characteristic (ROC) analysis. $P<0.05$ was considered statistically significant.

\section{Results}

\section{The baseline characteristics of the two groups}

There were 126 healthy individuals in the control group, with 103 individuals (83.7\%) were women. There were 73 cases with unilateral IJV hypoplasia in the study 
group, with 46 cases $(63.0 \%)$ were women. There was no difference of age between these two groups $(55.5 \pm 13.8$ years vs. $57.1 \pm 13.5$ years, $t=0.795, P=0.428$ ). The main symptoms of study groups were tinnitus cerebri (45 cases, 61.6\%), tinnitus (14 cases, 19.2\%), headache (10 cases, $13.7 \%$ ) and others (4 cases, 5.5\%). The median time for the symptoms duration were two months (interquartile range 1-7 months).

\section{The comparison of diameter and flow volume between the two groups}

In control groups, the diameter and $\mathrm{FVm}$ of on the left IJV were smaller than that of right IJV $(P<0.001$, Table 1). There were no difference of diameter ratio and
$\mathrm{FVm}$ ratio either in control group or in the study group (data not shown). In the study group, there were 67 cases (91.8\%) were with left IJV hypoplasia and 6 cases were with right IJV hypoplasia. The diameter and FVm of all three segments on the hypoplasia side were significantly lower than that on the dominant side, and were lower than the parameters of left (non-dominant) side of control group $(P<0.001$, Table 1$)$. The diameter of $\mathrm{J} 1$ and FVm of all three segments of IJV on the dominant side in study group were obviously higher than that of the right (dominant) side in control group, suggesting the compensation of dominant IJV $(P<0.05$, Table 1). Fig 1 showed the ultrasound images and MRV images of a patient with unilateral IJV hypoplasia.

Table 1 Bilateral J1 diameter and blood flow volume of the two groups

\begin{tabular}{|c|c|c|c|c|c|c|c|c|c|c|}
\hline \multirow[b]{2}{*}{ Parameter } & \multicolumn{5}{|c|}{ Control group } & \multicolumn{5}{|c|}{ Study group } \\
\hline & Right & Left & Left/Right & $t$ value & $P$ value & Dominant & Hypoplasia & $\begin{array}{c}\text { Hypoplasia/ } \\
\text { Dominant }\end{array}$ & $t$ value & $P$ value \\
\hline Diameter (mm) & $9.29 \pm 1.32$ & $8.35 \pm 1.05$ & $0.91 \pm 0.17$ & 6.697 & $<0.001$ & $9.97 \pm 1.26^{\mathrm{a}}$ & $6.33 \pm 1.40^{\mathrm{b}}$ & $0.65 \pm 0.17^{\mathrm{c}}$ & 16.599 & $<0.001$ \\
\hline $\mathrm{FVm}(\mathrm{ml} / \mathrm{min})$ & $926 \pm 252$ & $764 \pm 229$ & $0.86 \pm 0.39$ & 5.604 & $<0.001$ & $1235 \pm 397^{\mathrm{a}}$ & $354 \pm 217^{\mathrm{b}}$ & $0.30 \pm 0.18^{\mathrm{c}}$ & 18.240 & $<0.001$ \\
\hline
\end{tabular}

Note: FVm: mean flow volume. a: compared with the right side of control group, $P<0.05$, b: compared with the left side of control group, $P<0.05$. c: compared with the left/right ratio of control group, $P<0.001$.
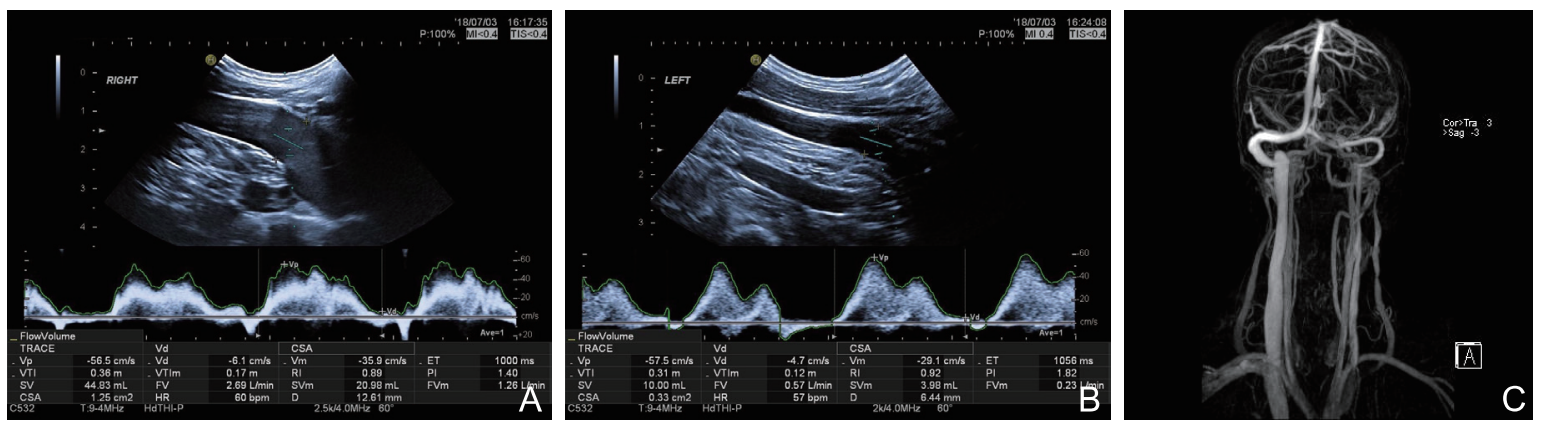

Figure 1 Ultrasound and MRV IJV images of a 55-year-old man A, The spectrum of right J1 with a diameter $12.61 \mathrm{~mm}$ and FVm $1.26 \mathrm{~L} / \mathrm{min} \mathrm{B}$, The spectrum of left J1 with a diameter $6.44 \mathrm{~mm}$ and FVm $0.23 \mathrm{~L} / \mathrm{min} \mathrm{C}$, MRV image showed the left transverse sinus and sigmoid sinus stenosis and left IJV hypoplasia.

In study group, the $\mathrm{J} 1$ diameter ratio between the hypoplasia side to dominant side were linear correlation with the J1 FVm ratio, with a coefficient $0.720(P<$ 0.001, Fig. 2). The linear regression equation was: FVm ratio $=0.794 \times$ diameter ratio- 0.212 .

\section{The optimal cut-off values of the diameters and flow volumes of IJV hypoplasia by ROC analysis}

Fig 3 showed the ROC curves of J1-J3 diameter (with the area under the curve AUC 0.826-0.887) and FVm (with the AUC 0.904-0.960) (all $P<0.05$ ). Then the optimal cut-off values of diameters and FVm for identifying IJV hypoplasia were determined, with the diameter ratio (hypoplasia/dominant side) of $\mathrm{J} 1-\mathrm{J} 3$ segment $0.70,0.80,0.75$ respectively and with the $\mathrm{FVm}$

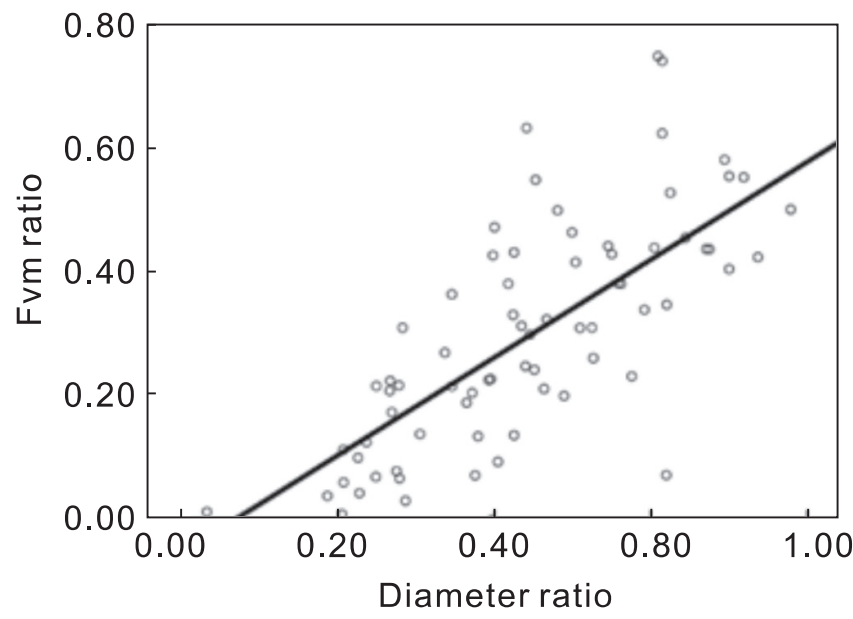

Figure 2 The linear correlation of the $\mathrm{J} 1$ diameter ratio and $\mathrm{FVm}$ ratio. 
ratio (hypoplasia/dominant side) of all three segments 0.50. In addition, the accuracy of FVm ratio was higher than that of diameters ratio (Table 3 ).

\section{The correlation of IJV hypoplasia and cerebral venous sinus stenosis}

In 67 cases with left IJV hypoplasia, there were 45 cases $(67.2 \%)$ with left side transverse sinus and/ or sigmoid sinus stenosis. In 6 cases with right IJV hypoplasia, there were 5 cases $(83.3 \%)$ with right side transverse sinus and/or sigmoid sinus stenosis. The side of IJV hypoplasia were highly correlated with the side of transverse sinus and/or sigmoid sinus stenosis, with an overall coincidence rate of $68.5 \%$ (50/73 cases). However, the diameter ratio and flow volume ratio of IJV hypoplasia side/dominant side had no differences between the two subgroups with and without cerebral venous sinus stenosis (all $P>0.05$, Table 4).

Table 2 The AUC of diameter ratio and FVm ratio (hypoplasia/dominant) of IJV hypoplasia

\begin{tabular}{|c|c|c|c|c|c|c|c|c|}
\hline \multirow{2}{*}{ Parameter } & \multicolumn{4}{|c|}{ Diameter ratio } & \multicolumn{4}{|c|}{ FVm ratio } \\
\hline & AUC & $95 \% \mathrm{CI}$ & SE & P value & AUC & $95 \% \mathrm{CI}$ & SE & $P$ value \\
\hline $\mathrm{J} 1$ & 0.871 & $0.820-0.922$ & 0.026 & $<0.001$ & 0.960 & $0.936-0.94$ & 0.012 & $<0.001$ \\
\hline $\mathrm{J} 2$ & 0.826 & $0.765-0.887$ & 0.031 & $<0.001$ & 0.904 & $0.864-0.945$ & 0.021 & $<0.001$ \\
\hline
\end{tabular}

Note: FVm: mean flow volume.

Table 3 The optimal cut-off values of diameter ration and FVm ratio for identifying IJV hypoplasia

\begin{tabular}{|c|c|c|c|c|c|c|c|c|}
\hline \multirow{2}{*}{ Parameter } & \multicolumn{4}{|c|}{ Diameter ratio } & \multicolumn{4}{|c|}{ FVm ratio } \\
\hline & Cut-off value & Sensitivity (\%) & Specificity (\%) & Youden,s index & Cut-off value & Sensitivity (\%) & Specificity (\%) & Youden,s index \\
\hline $\mathrm{J} 1$ & 0.70 & 92.1 & 64.4 & 0.565 & 0.50 & 92.1 & 86.3 & 0.784 \\
\hline $\mathrm{J} 2$ & 0.80 & 80.2 & 72.6 & 0.528 & 0.50 & 77.0 & 82.2 & 0.592 \\
\hline $\mathrm{J} 3$ & 0.75 & 81.7 & 82.2 & 0.639 & 0.50 & 84.1 & 94.5 & 0.786 \\
\hline
\end{tabular}

Note: FVm: mean flow volume.

Table 4 The diameter ratio and FVm ratio of IJV between subgroups with and without cerebral venous sinus stenosis

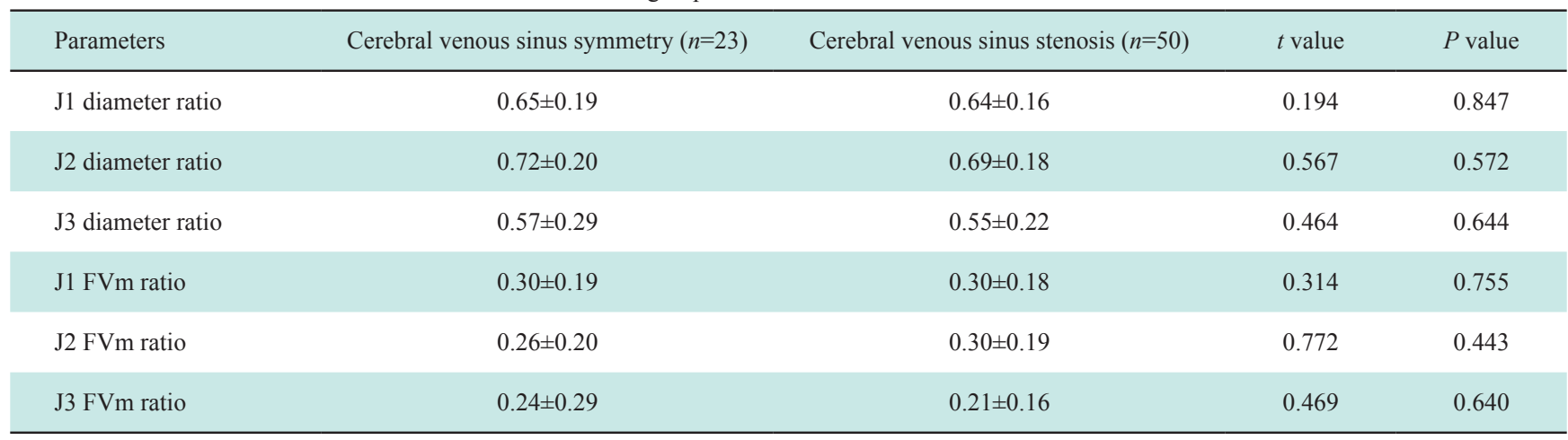

\section{Discussion}

The present study indicated that the diameter and $\mathrm{FVm}$ of right side were higher than the left side in healthy control group. In addition, in study group, most of the cases $(93.8 \%)$ of the IJV hypoplasia were on the left side. These findings showed that the right IJV was the dominant side, which were consistent with the previous studies [10-12]. The outflow difference between the bilateral IJV related to its anatomic variations: (1) the left side of IJV-innominate veinsuperior vena cava pathway is longer than the right side. Moreover, the angle of innominate vein influxes into the superior vena cava on the left side is larger than that on the right side. These anatomy characteristics determine that the outflow pathway on the right side is much smoother than the left side. (2) In healthy population, the sizes of bilateral jugular foreman were 
asymmetry. Freitas et al reported that in 30 autopsy samples, $73.3 \%$ right jugular foreman size was larger than the left side [13]. 3) IJV size was related to the variation of the superior sagittal sinus influx into

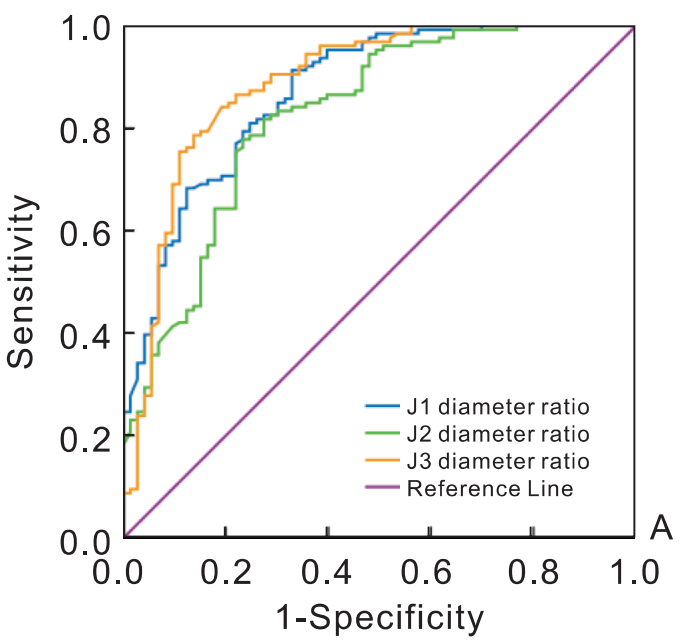

transverse sinus. Saiki et al found that $73.6 \%$ superior sagittal sinus influx into the right transverse sinus, meanwhile, the size of right transverse sinus and IJV was larger than that of the left side [14].

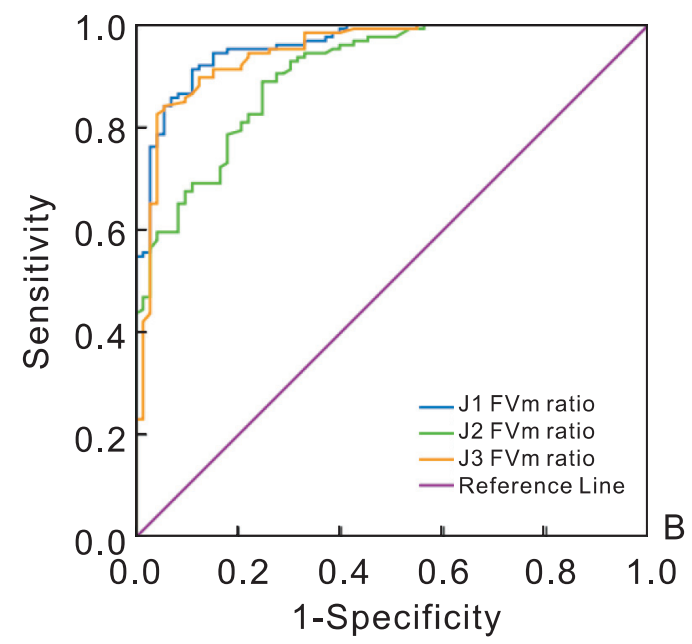

Figure 3 The ROC curves of J1、J2、J3 diameter ratio (A) and FVm ratio (B) for identifying IJV hypoplasia.

In this study, the diameter ratio and $\mathrm{FVm}$ ratio of the study group were significantly lower than the control group, which contributed to the symptoms of cerebral venous insufficiency. The mechanism of cerebral venous insufficiency induced clinical symptoms might be it induced the venous sinus pressure increase and leaded to the turbulence of venous flow [15]. Moreover, the diameter and FVm of dominant IJV in study group were higher than that of right (dominate) IJV in the control group, suggesting the compensation of the morphology and hemodynamics existed.

The main findings of this study was that established the optimal cut-off values of diameter ratio and flow volume ratio for identifying IJV hypoplasia. The hypoplasia/dominant ratio of diameter and flow volume were used in order to avoid the individual variant. The optimal cut-off diameter ratio of J1-J3 hypoplasia/ dominant side were $0.70,0.80$ and 0.75 respectively and the optimal cut-off flow volume ratio of three segments were all 0.50 . In addition, a linear regression equation (J1 $\mathrm{FVm}$ ratio $=0.794 \times$ diameter ratio-0.212) was established. According to this equation, the FVm of the hypoplasia side would be $50 \%$ of the contralateral side when the diameter ratio was 0.896 . However, the $\mathrm{FVm}$ ratio would be only 0.185 when the diameter ratio was 0.5 . Recently, West et al found that $30-35 \%$ stenosis of transverse sinus would lead to symptomatic intracranial hypertension [16]. These findings suggested that the venous hemodynamic parameter $(\mathrm{FVm})$ alteration was more sensitively than the morphology parameter (diameter), i.e. the smaller diameter alteration would lead to obvious hemodynamics change. The study also found out the accuracy and AUC of FVm ratio was higher than that of the diameter ratio for identifying hypoplasia. Thus, when evaluating hypoplasia, physicans should pay more attention to the hemodynamic alteration. It is known that the rate of diameter stenosis $\geqslant 50 \%$ could induce significant hemodynamic alteration in artery system. These difference of relationships of morphology and hemodynamics between artery and venous system might relate to the difference of vessel wall constituent, with the venous had thinner smooth muscle cells and lower compliance and elasticity [17].

Furthermore, the side of IJV hypoplasia were highly correlated with the side of transverse sinus and/or sigmoid sinus stenosis, which was consistent with the findings of Chao's study [17]. Chao et al reported that IJV hypoplasia was related to transverse sinus stenosis. When the $\mathrm{J} 3$ dominant/hypoplasia area ratio was 1.55 (i.e. the hypoplasia/dominant area ratio 0.64 or the diameter ratio 0.80 ), it can predict the transverse sinsus hypoplasia [7]. The cut-off values of this present study (J3 diameter ratio cut-off value 0.75 ) was similar with that. It should be noted that there were still $31.5 \%$ cases in study group with the bilateral cerebral venous sinus symmetry and the diameter ratio and $\mathrm{FVm}$ ratio of IJV hypoplasia side/dominant side had no differences between the two subgroups with and without cerebral venous sinus stenosis. Zhou et al demonstrated that only IJV stenosis (without venous sinus stenosis) would lead to intracranial hypertension [3]. Thus, when there was IJV hypoplasia or stenosis, it is still necessary to use MRV/CTV to 
examine the cerebral venous sinus in order to develop an integral therapeutic regimen.

For imaging evaluation IJV hypoplasia, physicians should pay more attention to the hemodynamics evaluation besides the morphology difference, including the contralateral compensation ability. As demonstrated in this study, the accuracy of FVm ratio was higher than that of the diameter ratio for identifying hypoplasia. Ultrasound can evaluate both the morphology and hemodynamics of IJV, which is the advantage of ultrasound. In addition, as a real-time, dynamic modality, ultrasound can view alteration of diameter and flow volume along with the respiration, which is the advantage of ultrasound over CTV or MRV. The advantage of CTV and MRV are they can evaluate the IJV and the cerebral venous sinus morphology at the same time.

The limitation of this study was it was a relatively small sample thus sub-analysis on age was absent. However, there was no difference on the age between study group and control group. In addition, diameter ratio and $\mathrm{FVm}$ ratio were used to minimize the individual variations. In the coming project, the venous sinus stenosis cases with and without IJV hypoplasia will be recruited to compare the parameters pre- and post treatment (cerebral venous intervention and/or IJV intervention) and further demonstrate the correlation mechanism of IJV hypoplasia and cerebral venous sinus stenosis.

\section{Acknowledgement}

This study was supported by the Chinese National Natural Science Foundation (81200912).

\section{Conflict of Interest}

The authors have no conflict of interest to declare.

\section{References}

[1] Tong LS, Guo ZN, Ou YB, Yu YN, Zhang XC, Tang J, et al. Cerebral venous collaterals: A new fort for fighting ischemic stroke? Prog Neurobiol 2018;163-164:172-193.

[2] Jia LY, Hua Y, Ji XM,Liu JT. Correlation analysis of internal jugular vein abnormalities and cerebral venous sinus thrombosis. Chinese Medical Journal 2012;125:3671-3674. [in Chinese]

[3] Zhou D, Meng R, Zhang X, Guo L, Li S, Wu W, et al. Intracranial hypertension induced by internal jugular vein stenosis can be resolved by stenting. Eur J Neurol 2018;25:365-e13.

[4] Han K, Chao AC, Chang FC, Chung CP, Hsu HY, Sheng WY, et al Obstruction of venous drainage linked to transient global amnesia. PLoS One 2015;10:e132893.

[5] Hsu HY, Chao AC, Chen YY, Yang FY, Chung CP, Sheng WY, et al. Reflux of jugular and retrobulbar venous flow in transient monocular blindness. Ann Neurol 2008;63:247-253.

[6] Sethi SK, Daugherty AM, Gadda G, Utriainen DT, Jiang J, Raz $\mathrm{N}$, et al. Jugular anomalies in multiple sclerosis are associated with increased collateral venous flow. AJNR Am J Neuroradiol 2017;38:1617-1622.

[7] Dai YF, Li KC, Zhang KY, Liang PP. Administration method of contrast agent for large field of view 3D high-spatial-resolution contrast-enhanced MRV of cerebral venous sinus and jugular veins. Chin J Med Imaging Technol 2014;30:126-129.[in Chinese]

[8] Zaharchuk G, Fischbein NJ, Rosenberg J, Herfkens RJ, Dake MD. Comparison of MR and contrast venography of the cervical venous system in multiple sclerosis. AJNR Am J Neuroradiol 2011;32:1482 1489.

[9] Han K, Chao AC, Chang FC, Hsu HY, Chung CP, Sheng WY, et al. Diagnosis of transverse sinus hypoplasia in magnetic resonance venography: new insights based on magnetic resonance imaging in combined dataset of venous outflow impairment case-control studies: post hoc case-control study. Medicine (Baltimore) 2016;95:e2862.

[10] Laganà MM, Pelizzari L, Scaccianoce E, Dipasquale O, Ricci C, Baglio F, et al. Assessment of internal jugular vein size in healthy subjects with magnetic resonance and semiautomatic processing. Behav Neurol 2016;2016:9717210.

[11] Chambers B, Chambers J, Churilov L, Cameron H, Macdonell R. Internal jugular and vertebral vein volume flow in patients with clinically isolated syndrome or mild multiple sclerosis and healthy controls: results from a prospective sonographer-blinded study. Phlebology 2014;29:528-535.

[12] Chung CP, Lin YJ, Chao AC, Lin SJ, Chen YY, Wang YJ, et al. Jugular venous hemodynamic changes with aging. Ultrasound Med Biol 2010;36:1776-1182.

[13] Freitas CAF, Santos LRMD, Santos AN, Amaral Neto ABD, Brandão LG. Anatomical study of jugular foramen in the neck. Braz $J$ Otorhinolaryngol 2018; pii: S1808-8694(18)30715-30718.

[14] Saiki K, Tsurumoto T, Okamoto K, Wakebe T. Relation between bilateral differences in internal jugular vein caliber and flow patterns of dural venous sinuses. Anat Sci Int 2013;88:141-150.

[15] Markey KA, Mollan SP, Jensen RH, Sinclair AJ.Understanding idiopathic intracranial hypertension: mechanisms, management, and future directions. Lancet Neurol 2016;15:78-91.

[16] West JL, Greeneway GP, Garner RM, Aschenbrenner CA, Singh J, Wolfe SQ, et al. Correlation between angiographic stenosis and physiologic venous sinus outflow obstruction in idiopathic intracranial hypertension. J Neurointerv Surg 2019;11:90-94

[17] Chao AC, Han K, Chang FC, Hsu HY, Chung CP, Sheng WY, et al. Ultrasound diagnosis of transverse sinus hypoplasia using flow profiles of the internal jugular vein. PLoS One 2017;12:e0181119. 